ANNALES

POLONICI MATHEMATICI

$93.2(2008)$

\title{
Starlikeness of polynomials and finite Blaschke products
}

\author{
by Alan Gluchoff and Frederick Hartmann (Villanova, PA)
}

\begin{abstract}
The radius of starlikeness for polynomial mappings and finite Blaschke products with zeroes distributed at equal angles around a circle centered at the origin, as well as with zeroes concentrated at a single point, are considered, and sharp bounds are obtained. Results expressing the radius of starlikeness of an arbitrary polynomial or Blaschke product in terms of the magnitudes of the zeroes are also given. These are also sharp.
\end{abstract}

1. Introduction. Let $f(z)=a_{1} z+a_{2} z^{2}+\cdots$ be analytic on the unit disk $D=\{z:|z|<1\}$ of the complex plane, and let $r<1$. The question of the radius of starlikeness of $f$, that is, the largest value of $r$ for which $D_{r}=\{z:|z|<r\}$ is mapped in a one-to-one manner onto a region starlike with respect to the origin, has been a question of interest beginning with [1], in which the author considered primarily polynomial mappings. Others contributed much to the calculation of $r$ and related radii of univalence and convexity for polynomials, sometimes expressing the values in terms of the zeroes and critical points; [2] contains a survey of this work. These results appear to deal mainly with a single configuration of the zeroes, namely that in which the nontrivial zeroes are concentrated at a single point.

In this paper we deal with the case of polynomials and finite Blaschke products whose zeroes are distributed at equal angles around a circle centered at the origin of radius less than 1 . Even for these relatively straightforward configurations the calculation of $r$ can be quite complicated. We give sharp upper and lower bounds on $r$ for these cases, and compare the values for polynomials and finite Blaschke products. We also give other results for an arbitrary polynomial and Blaschke product which express $r$ in terms of the magnitudes of the nontrivial zeroes; these bounds are also sharp.

2000 Mathematics Subject Classification: Primary 30C45; Secondary 30C10, 30D50.

Key words and phrases: univalent functions, starlike poynomials, starlike Blaschke products. 
2. Radius of starlikeness for polynomials. Define the radius of starlikeness for $f(z)$ to be the largest value of $r$ for which the disk $D_{r}=\{z$ : $|z|<r\}$ is mapped one-to-one to a starlike region by $f$. We assume that the polynomial $P_{n}(z)$ has $n$ zeroes located on the circle $\{z:|z|=\varrho, 0<\varrho \leq 1\}$ and a single zero at the origin and write

$$
P_{n}(z)=z \prod_{k=1}^{n}\left(1-\frac{z}{z_{k}}\right)=z \prod_{k=1}^{n}\left(1-\frac{z}{\varrho e^{i \theta_{k}}}\right) .
$$

The polynomial $P_{n}$ maps $D_{r}=\{z:|z|<r\}$ one-to-one to a starlike region if and only if $\Re\left\{z P_{n}^{\prime}(z) / P_{n}(z)\right\}>0$ for $z \in D_{r}$. Using logarithmic differentiation one has

$$
\Re\left\{\frac{z P_{n}^{\prime}(z)}{P_{n}(z)}\right\}=1+\Re\left\{\sum_{k=1}^{n} \frac{z}{z-z_{k}}\right\} .
$$

One can easily compute, using (1), the radius of starlikeness for two extreme cases.

FACT 1. Let $P_{n}(z)=z(1-z / \varrho)^{n}$ (i.e. $P_{n}(z)$ has all of its nontrivial zeroes at the point $z=\varrho)$. Then the radius of starlikeness for $P_{n}$ is $\varrho /(n+1)$.

FACT 2. Let $P_{n}(z)=z\left(1-z^{n} / \varrho^{n}\right)$, (i.e. $P_{n}(z)$ has its nontrivial zeroes evenly distributed about the circle of radius $\varrho)$. Then the radius of starlikeness for $P_{n}$ is $\varrho(n+1)^{-1 / n}$.

We require the following three technical lemmas:

LEMma 1. $\phi(r)=r /(r-\varrho), 0<r \leq \varrho \leq 1$, is a decreasing function of $r$.

Proof. We have

$$
\frac{\partial \phi(r)}{\partial r}=-\frac{\varrho}{(r-\varrho)^{2}}
$$

LEMma 2. The function

$$
\phi(r)=\frac{r}{r-\varrho}-\frac{r \varrho}{r \varrho-1}, \quad 0<r \leq \varrho \leq 1,
$$

is a decreasing function of $r$.

Proof. Indeed,

$$
\frac{\partial \phi(r)}{\partial r}=-\frac{\varrho\left(1-r^{2}\right)\left(1-\varrho^{2}\right)}{(\varrho-r)^{2}(r \varrho-1)^{2}}
$$

Lemma 3. For $n=1,2, \ldots$ and $0<r<\varrho \leq 1$ one has

$$
\frac{r^{n}}{r^{n}-\varrho^{n}}-\frac{r^{n} \varrho^{n}}{r^{n} \varrho^{n}-1} \geq \frac{r}{r-\varrho}-\frac{r \varrho}{r \varrho-1} .
$$


Proof. For $n=1$ the statement is an identity. The case $n=2$ follows from the identity

$$
\frac{r^{2}}{r^{2}-\varrho^{2}}-\frac{r^{2} \varrho^{2}}{r^{2} \varrho^{2}-1}-\frac{r}{r-\varrho}+\frac{r \varrho}{r \varrho-1}=\frac{\varrho r\left(r^{2}+1\right)(\varrho-1)(\varrho+1)}{(r \varrho-1)(\varrho-r)(r \varrho+1)(\varrho+r)} .
$$

For $n \geq 3$ and $0<r<\varrho \leq 1$ consider

$$
\begin{aligned}
& \frac{r^{n} \varrho^{n}}{1-r^{n} \varrho^{n}}-\frac{r^{n}}{\varrho^{n}-r^{n}} \geq \frac{r \varrho}{1-r \varrho}-\frac{r}{\varrho-r} \\
& \Leftrightarrow \frac{r^{n}\left(\varrho^{2 n}-1\right)}{\left(1-r^{n} \varrho^{n}\right)\left(\varrho^{n}-r^{n}\right)} \geq \frac{r\left(\varrho^{2}-1\right)}{(1-r \varrho)(\varrho-r)} \\
& \Leftrightarrow \frac{r^{n}\left(\varrho^{2 n}-1\right)}{\left(1-r^{n} \varrho^{n}\right)\left(\varrho^{n}-r^{n}\right)} \geq \frac{r\left(\varrho^{2}-1\right) \sum_{k=0}^{n-1}(r \varrho)^{k} \sum_{k=0}^{n-1} r^{k} \varrho^{n-k-1}}{\left[(1-r \varrho) \sum_{k=0}^{n-1}(r \varrho)^{k}\right]\left[(\varrho-r) \sum_{k=0}^{n-1} r^{k} \varrho^{n-k-1}\right]} \\
& \Leftrightarrow \frac{r^{n}\left(\varrho^{2 n}-1\right)}{\left(1-r^{n} \varrho^{n}\right)\left(\varrho^{n}-r^{n}\right)} \geq \frac{r\left(\varrho^{2}-1\right) \sum_{k=0}^{n-1}(r \varrho)^{k} \sum_{k=0}^{n-1} r^{k} \varrho^{n-k-1}}{\left(1-r^{n} \varrho^{n}\right)\left(\varrho^{n}-r^{n}\right)} \\
& \Leftrightarrow r^{n}\left(\varrho^{2 n}-1\right) \geq r\left(\varrho^{2}-1\right) \sum_{k=0}^{n-1}(r \varrho)^{k} \sum_{k=0}^{n-1} r^{k} \varrho^{n-k-1} \\
& \Leftrightarrow r^{n}\left(\varrho^{2}-1\right)\left(\sum_{k=0}^{n-1} \varrho^{2 k}\right)-r\left(\varrho^{2}-1\right) \sum_{k=0}^{n-1}(r \varrho)^{k} \sum_{k=0}^{n-1} r^{k} \varrho^{n-k-1} \geq 0 \\
& \Leftrightarrow r\left(\varrho^{2}-1\right)\left[r^{n-1} \sum_{k=0}^{n-1} \varrho^{2 k}-\sum_{k=0}^{n-1}(r \varrho)^{k} \sum_{k=0}^{n-1} r^{k} \varrho^{n-k-1}\right] \geq 0 \\
& \Leftrightarrow r\left(\varrho^{2}-1\right)\left[r^{n-1} \sum_{k=0}^{n-1} \varrho^{2 k}+r^{n-1}-\left[\sum_{k=0}^{n-1}(r \varrho)^{k}\right] r^{n-1}-\sum_{k=0}^{n-1}(r \varrho)^{k} \sum_{k=0}^{n-2} r^{k} \varrho^{n-k-1}\right] \geq 0 \\
& \Leftrightarrow r\left(\varrho^{2}-1\right)\left[r^{n-1} \sum_{k=0}^{n-1} \varrho^{2 k}-\left[\sum_{k=1}^{n-1}(r \varrho)^{k}\right] r^{n-1}-\sum_{k=0}^{n-1}(r \varrho)^{k} \sum_{k=0}^{n-2} r^{k} \varrho^{n-k-1}\right] \geq 0 \\
& \Leftrightarrow r \varrho\left(\varrho^{2}-1\right)\left[r^{n-1} \sum_{k=0}^{n-1} \varrho^{2 k-1}-\sum_{k=1}^{n-1} r^{k} \varrho^{k-1} r^{n-1}-\sum_{k=0}^{n-1}(r \varrho)^{k} \sum_{k=0}^{n-2} r^{k} \varrho^{n-k-2}\right] \geq 0 .
\end{aligned}
$$

Now every term in the first sum (within the square brackets) is canceled by a term in the product of two sums, leaving only a negative expression within the brackets. This gives the desired result.

The following theorem can be traced back to [1]; several authors have discovered more modern proofs (see [2, p. 10] for references). We include the theorem for the sake of completeness.

Theorem 1. Let $P_{n}(z)=z \prod_{k=1}^{n}\left(1-z / \varrho e^{i \theta_{k}}\right)$ and $r<r_{\min }:=\varrho /(n+1)$. Then $P_{n}$ maps the disk of radius $r$ about the origin univalently to a starlike region. 
Proof. We have

$$
\begin{aligned}
0 & =1+n \frac{r_{\min }}{r_{\min }-\varrho}=1+\sum_{k=1}^{n} \frac{r_{\min }}{r_{\min }-\varrho} \\
& <1+\sum_{k=1}^{n} \frac{r}{r-\varrho}=1+\sum_{k=1}^{n} \min _{t \in[0,2 \pi]} \Re\left\{\frac{r e^{i t}}{r e^{i t}-\varrho e^{i \theta_{k}}}\right\} \leq\left.\Re\left\{\frac{z P_{n}^{\prime}(z)}{P_{n}(z)}\right\}\right|_{z=r e^{i t}} .
\end{aligned}
$$

Hence $P_{n}(z)$ is starlike on the disk of radius $r$.

ThEOREM 2. Let $P_{n}(z)=z \prod_{k=1}^{n}\left(1-z / \varrho e^{i \theta_{k}}\right)$ and $r>r_{\max }:=\varrho(n+1)^{-1 / n}$. Then $P_{n}$ maps the disk of radius $r$ about the origin to a nonstarlike region.

Proof. We have

$$
\begin{aligned}
0 & =1+n \frac{r_{\max }^{n}}{r_{\max }^{n}-\varrho^{n}}>1+n \frac{r_{\max }}{r_{\max }-\varrho} \quad \text { [Lemma 1] } \\
& >1+n \frac{r}{r-\varrho}=1+\sum_{k=1}^{n} \min _{t \in[0,2 \pi]} \Re\left\{\frac{r e^{i t}}{r e^{i t}-\varrho e^{i \theta_{k}}}\right\} \quad \text { [Lemma 1] } \\
& =\left.\min _{t \in[0,2 \pi]} \Re\left\{\frac{z P_{n}^{\prime}(z)}{P_{n}(z)}\right\}\right|_{z=r e^{i t}} .
\end{aligned}
$$

Hence $P_{n}$ fails to map the disc of radius $r$ about the origin univalently to a starlike region.

COROLlaRy 1. The radius of starlikeness, $r$, for $P_{n}(z)$ satisfies

$$
\frac{\varrho}{n+1} \leq r \leq \frac{\varrho}{(n+1)^{1 / n}}
$$

and this result is sharp.

It is an interesting but difficult problem to find the radius of starlikeness as a function of the distribution of the zeroes on the circle of radius $\varrho$, even for a polynomial with $n=2$. We consider the case of two zeroes on the circle of radius $\varrho$ and without loss of generality may assume that the zeroes are $z_{1}=\varrho e^{i \theta}$ and $z_{2}=\varrho e^{-i \theta}$. Then

$$
P_{2}(z)=z\left(1-\frac{z}{\varrho e^{i \theta}}\right)\left(1-\frac{z}{\varrho e^{-i \theta}}\right)=z\left(z^{2}-2 \varrho \cos (\theta) z+\varrho^{2}\right) .
$$

Theorem 3. Let $P_{2}(z)=z\left(z^{2}-2 \varrho \cos (\theta) z+\varrho^{2}\right)$. Then the radius of starlikeness, $r(\theta, \varrho)$, of $P_{2}(z)$ is given by

$$
r(\theta, \varrho)= \begin{cases}\varrho\left(\frac{2}{3} \cos (\theta)-\frac{1}{3} \sqrt{4 \cos ^{2}(\theta)-3}\right) & \text { if } 0 \leq \theta<\arccos \left(\frac{2}{5} \sqrt{5}\right) \\ \varrho \sqrt{\frac{9 \cos ^{2}(\theta)-8}{25 \cos ^{2}(\theta)-24}} & \text { if } \arccos \left(\frac{2}{5} \sqrt{5}\right) \leq \theta \leq \pi / 2 .\end{cases}
$$


Proof. We have

(2) $\left.\Re\left\{\frac{z P_{2}^{\prime}(z)}{P_{2}(z)}\right\}\right|_{z=r e^{i t}}=$

$\frac{8 r^{2} \varrho^{2} \cos ^{2}(t)+3 r^{4}-\left(10 r^{3} \varrho+6 r \varrho^{3}\right) \cos (\theta) \cos (t)+8 r^{2} \varrho^{2} \cos ^{2}(\theta)+\varrho^{4}-4 r^{2} \varrho^{2}}{4 r^{2} \varrho^{2} \cos ^{2}(t)+r^{4}-\left(4 r^{3} \varrho+4 r \varrho^{3}\right) \cos (\theta) \cos (t)+4 r^{2} \varrho^{2} \cos ^{2}(\theta)+\varrho^{4}-2 r^{2} \varrho^{2}}$.

(2) has a global minimum of zero at an interior point of $[0,2 \pi]$ if $\arccos \left(\frac{2}{5} \sqrt{5}\right) \leq \theta \leq \pi / 2$, in which case

$$
r=\varrho \sqrt{\frac{9 \cos ^{2}(\theta)-8}{25 \cos ^{2}(\theta)-24}}
$$

(2) has a global minimum of zero at $t=0$ and $t=2 \pi$ if $0 \leq \theta<\arccos \left(\frac{2}{5} \sqrt{5}\right)$, in which case

$$
r=\varrho\left(\frac{2}{3} \cos (\theta)-\frac{1}{3} \sqrt{4 \cos ^{2}(\theta)-3}\right)
$$

Figure 1 shows the radius of starlikeness a function of $\theta \in[0,2 \pi]$ for $\varrho=1$. Even the case of three zeroes on the circle of radius $\varrho$ for a polynomial with real coefficients presents an intractable problem. Without loss of generality we may assume that $P_{3}(z)=z(1-z)\left(1-z / \varrho e^{-i \theta}\right)\left(1-z / \varrho e^{i \theta}\right)$. We have obtained an implicit relation between $r$ and $\theta$, but have been unable to find an explicit functional relation from this implicit form like that in Theorem 3. Numerical and graphical calculations do, however, confirm the results of Theorems 1 and 2 .

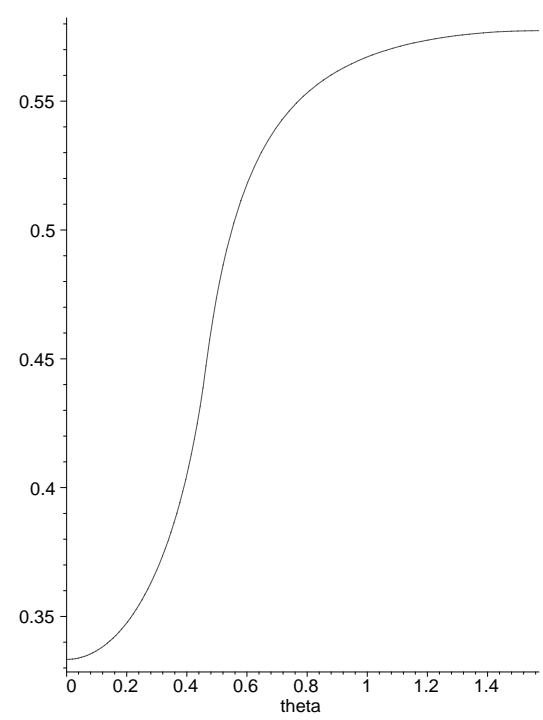

Fig. 1 
A theorem for a more general kind of polynomials giving a sufficient condition for radius of starlikeness is:

ThEOREM 4. Let $P_{n}(z)=z \prod_{k=1}^{n}\left(1-z / z_{k}\right)$ where $z_{k}=\varrho_{k} e^{i \theta_{k}}$ and $0<\varrho_{1} \leq \cdots \leq \varrho_{n}$. Then $P_{n}(z)$ maps $D_{r}=\{z:|z|<r\}$ univalently onto a starlike region when

$$
r<\frac{\varrho_{1}}{1+\sum_{k=1}^{n} \varrho_{1} / \varrho_{k}} .
$$

Proof. We have $\Re\left[z P_{n}^{\prime}(z) / P_{n}(z)\right]=1+\sum_{k=1}^{n} \Re\left[z /\left(z-z_{k}\right)\right]$ and if $z=r e^{i t}$, $z_{k}=\varrho_{k} e^{i \theta_{k}}, 0<\varrho_{1} \leq \cdots \leq \varrho_{n}$, then

$$
1+\sum_{k=1}^{n} \Re\left[\frac{z}{z-z_{k}}\right]>1+\sum_{k=1}^{n} \frac{r}{r-\varrho_{k}}=1-\sum_{k=1}^{n} \frac{r / \varrho_{k}}{1-r / \varrho_{k}} .
$$

But $r / \varrho_{k} \leq r / \varrho_{1}$, which implies $\left(r / \varrho_{k}\right) /\left(1-r / \varrho_{k}\right) \leq\left(r / \varrho_{k}\right) /\left(1-r / \varrho_{1}\right)$. Therefore

$$
\Re\left[\frac{z P_{n}^{\prime}(z)}{P_{n}(z)}\right] \geq 1-\sum_{k=1}^{n} \frac{r / \varrho_{k}}{1-r / \varrho_{1}}=1-r \sum_{k=1}^{n} \frac{\varrho_{1} / \varrho_{k}}{\varrho_{1}-r} \geq 0
$$

if and only if $|z| \leq r<\varrho_{1} /\left(1+\sum_{k=1}^{n} \varrho_{1} / \varrho_{k}\right)$.

REMARK. If $\varrho_{1}=\cdots=\varrho_{n}$ then $\varrho_{1} /\left(1+\sum_{k=1}^{n} \varrho_{1} / \varrho_{k}\right)=\varrho_{1} /(n+1)$ so the result is sharp in the sense that there is a configuration of the $z_{k}$ 's for which $\varrho_{1} /\left(1+\sum_{k=1}^{n} \varrho_{1} / \varrho_{k}\right)$ gives the exact answer. [See Fact 1.]

3. Radius of starlikeness for finite Blaschke products. We consider finite Blaschke products of the following kind:

$$
B_{n}(z)=z \prod_{k=1}^{n} \frac{z-z_{k}}{1-\bar{z}_{k} z}
$$

where the $z_{k}$ 's lie in the unit disk. For sufficiently small $r, B_{n}$ maps $D_{r}=\{z$ : $|z|<r\}$ to a starlike region. The largest value of $r$ for which $D_{r}$ is mapped to a starlike region is called the radius of starlikeness for $B_{n}$. Logarithmic differentiation gives, for $\left|z_{k}\right| \leq 1$,

$$
\Re\left\{\frac{z B_{n}^{\prime}(z)}{B_{n}(z)}\right\}=1+\Re\left\{\sum_{k=1}^{n} \frac{z}{z-z_{k}}-\frac{z \bar{z}_{k}}{z \bar{z}_{k}-1}\right\} .
$$

We assume that $B_{n}(z)$ has $n$ zeroes located on the circle $\{z:|z|=\varrho, 0<$ $\varrho<1\}$ and a single zero at the origin and write

$$
B_{n}(z)=z \prod_{k=1}^{n} \frac{z-\varrho e^{i \theta_{k}}}{1-\varrho e^{-i \theta_{k} z}} .
$$

We consider as before two special cases: the case where there is a zero of order $n$ at the point $z=\varrho$ so that $B_{n}(z)=z\left(\frac{z-\varrho}{1-\varrho z}\right)^{n}$, and the case where 
there are $n$ zeroes evenly distributed around the circle of radius $\varrho$ so that $B_{n}(z)=z \frac{z^{n}-\varrho^{n}}{1-\varrho^{n} z^{n}}$. Using (4) one can obtain the following results:

FACT 3. If

$$
B_{n}(z)=z\left(\frac{z-\varrho}{1-\varrho z}\right)^{n}
$$

then the radius of starlikeness $r(\varrho, n)$ for $B_{n}(z)$ is

$$
r(\varrho, n)=\frac{1+\varrho^{2}+n\left(1-\varrho^{2}\right)-\sqrt{\left(1-\varrho^{2}\right)\left((n+1)^{2}-\varrho^{2}(n-1)^{2}\right)}}{2 \varrho} .
$$

FACT 4. If

$$
B_{n}(z)=z \frac{z^{n}-\varrho^{n}}{1-\varrho^{n} z^{n}}
$$

then the radius of starlikeness $r(\varrho, n)$ for $B_{n}(z)$ is

(6) $r(\varrho, n)$

$$
=\left(\frac{(1+n)+\varrho^{2 n}(1-n)-\sqrt{\varrho^{4 n}(n-1)^{2}-2 \varrho^{2 n}\left(1+n^{2}\right)+(n+1)^{2}}}{2 \varrho^{n}}\right)^{1 / n} .
$$

TheOREM 5. Let

$$
B_{n}(z)=z \prod_{k=1}^{n} \frac{z-\varrho e^{i \theta_{k}}}{1-\varrho e^{-i \theta_{k} z}}
$$

and $0<r \leq r^{*}$ where

$$
r^{*}=\frac{1+\varrho^{2}+n\left(1-\varrho^{2}\right)-\sqrt{\left(1-\varrho^{2}\right)\left((n+1)^{2}-\varrho^{2}(n-1)^{2}\right)}}{2 \varrho} .
$$

Then $B_{n}$ maps $D_{r}$ univalently onto a starlike region.

Proof. We have

$$
\begin{aligned}
0 & =1+\frac{n r^{*}}{r^{*}-\varrho}-\frac{n r^{*} \varrho}{r^{*} \varrho-1}=1+\sum_{k=1}^{n}\left(\frac{r^{*}}{r^{*}-\varrho}-\frac{r^{*} \varrho}{r^{*} \varrho-1}\right) \\
& \leq 1+\sum_{k=1}^{n}\left(\frac{r}{r-\varrho}-\frac{r \varrho}{r \varrho-1}\right) \quad[\text { Lemma } 2] \\
& =1+\sum_{k=1}^{n} \min _{t \in[0,2 \pi]} \Re\left\{\frac{r e^{i t}}{r e^{i t}-\varrho e^{i \theta_{k}}}-\frac{r e^{i t} \varrho e^{i \theta_{k}}}{r e^{i t} \varrho e^{i \theta_{k}}-1}\right\} \\
& =\left.\Re\left\{\frac{z B_{n}^{\prime}(z)}{B_{n}(z)}\right\}\right|_{z=r e^{i t}} .
\end{aligned}
$$

Theorem 6. Let $B_{n}(z)$ be as in Theorem 5 , and $r_{*}<r$ where $r_{*}=\left(\frac{(1+n)+\varrho^{2 n}(1-n)-\sqrt{\varrho^{4 n}(n-1)^{2}-2 \varrho^{2 n}\left(1+n^{2}\right)+(n+1)^{2}}}{2 \varrho^{n}}\right)^{1 / n}$.

Then $B_{n}$ fails to map $D_{r}$ univalently onto a starlike region. 
Proof. Indeed,

$$
\begin{aligned}
0 & =1+n \frac{r_{*}^{n}}{r_{*}^{n}-\varrho^{n}}-n \frac{r_{*}^{n} \varrho^{n}}{r_{*}^{n} \varrho^{n}-1}>1+n\left[\frac{r^{n}}{r^{n}-\varrho^{n}}-\frac{r^{n} \varrho^{n}}{r^{n} \varrho^{n}-1}\right] \text { [Lemma 2] } \\
& =1+\sum_{k=1}^{n}\left(\frac{r^{n}}{r^{n}-\varrho^{n}}-\frac{r^{n} \varrho^{n}}{r^{n} \varrho^{n}-1}\right) \geq 1+\sum_{k=1}^{n}\left(\frac{r}{r-\varrho}-\frac{r \varrho}{r \varrho-1}\right) \text { [Lemma 3] } \\
& =1+\sum_{k=1}^{n} \min _{t \in[0,2 \pi]} \Re\left\{\frac{r e^{i t}}{r e^{i t}-\varrho e^{i \theta_{k}}}-\frac{r e^{i t} \varrho e^{i \theta_{k}}}{r e^{i t} \varrho e^{i \theta_{k}}-1}\right\} \\
& =\left.\Re\left\{\frac{z B_{n}^{\prime}(z)}{B_{n}(z)}\right\}\right|_{z=r e^{i t}} .
\end{aligned}
$$

CoROllary 2. The radius of starlikeness $r$ for $B_{n}(z)$ satisfies $r^{*} \leq r$ $\leq r_{*}$ and this result is sharp.

There is a relation among the various radii of starlikeness just developed. It is a straightforward but tedious computation to show (see Theorems 1, 2, 5 and 6 for notation) that $r_{\min } \leq r_{*}$ and $r_{\max } \leq r^{*}$. Note: These inequalities are in accord with the condition for starlikeness of polynomials and Blaschke products given in [3]: for $B(z)$ as in (3) one knows that $B$ is starlike in $\{z:|z|<r\}$ if and only if

$$
\theta-\pi / 2<\arg \left[\frac{1}{\overline{r e^{i \theta}}-\overline{0}}+\sum_{k=1}^{n-1} \frac{1}{\overline{r e^{i \theta}}-\bar{z}_{k}}+\sum_{k=1}^{n-1} \frac{-1}{\overline{r e^{i \theta}}-\overline{z_{k}^{*}}}\right]<\theta+\pi / 2
$$

for all $\theta$, where $z=r e^{i \theta}, z_{k}^{*}=1 / \bar{z}_{k}$. For polynomials $P(z)=z \prod_{k=1}^{n-1}\left(1-\frac{z}{z_{k}}\right)$ the condition is the same except the second sum is omitted. The condition has the following physical interpretation: if unit masses are placed at the $z_{k}$ 's (including zero) with repulsive force at $r e^{i \theta}$ of magnitude $1 /\left|r e^{i \theta}-z_{k}\right|$, and unit masses are placed at the $z_{k}^{*}$ 's with attractive force of magnitude $1 /\left|r e^{i \theta}-z_{k}^{*}\right|$ at $r e^{i \theta}$, then the starlikeness of $B$ (or $P$ ) in $\{z:|z|<r\}$ is equivalent to the requirement that the sum of the forces directed at $r e^{i \theta}$ points in a direction outward from the boundary $|z|=r$ at any $\theta$. Thus the presence of $z_{k}^{*}$ makes it seem plausible that the radius of starlikeness for $B$ is larger than that of $P$.

A theorem of a more general nature for finite Blaschke products (see by comparison Theorem 4) is the following:

Theorem 7. Let $B_{n}(z)$ be as in (3) with $z_{k}=\varrho_{k} e^{i \theta_{k}}, G=\sqrt{\varrho_{1} \varrho_{n}}$ and $0<\varrho_{1} \leq \cdots \leq \varrho_{n}<1$. Then $B_{n}(z)$ is starlike univalent on the disk of radius $r$ where $r<r^{*}=2 \varrho_{1} /\left(A_{n}+\sqrt{B_{n}}\right)$, with

$$
A_{n}:=1+G^{2}+\sum_{k=1}^{n}\left(1-\varrho_{k}^{2}\right)\left(\frac{G}{\varrho_{k}}\right)^{2}
$$


and

$$
B_{n}:=\left[(1+G)^{2}+\sum_{k=1}^{n}\left(1-\varrho_{k}^{2}\right)\left(\frac{G}{\varrho_{k}}\right)^{2}\right]\left[(1-G)^{2}+\sum_{k=1}^{n}\left(1-\varrho_{k}^{2}\right)\left(\frac{G}{\varrho_{k}}\right)^{2}\right] .
$$

Proof. We have

$$
\Re\left[\frac{z B^{\prime}(z)}{B(z)}\right]=1+\sum_{k=1}^{n} \frac{\varrho^{k}-1}{\bar{z}_{k} z+z_{k} / z-\left(1+\varrho_{k}^{2}\right)} .
$$

Now $\bar{z}_{k} z+z_{k} / z$ maps $\{z:|z|=r\}$ to an ellipse with real axis intersections $-\varrho_{k}(r+1 / r)$ and $\varrho_{k}(r+1 / r)$ and it follows that

$$
\begin{aligned}
\Re\left[\frac{\varrho^{k}-1}{\bar{z}_{k} z+z_{k} / z-\left(1+\varrho_{k}^{2}\right)}\right] & \geq \frac{\varrho_{k}^{2}-1}{\varrho_{k}(r+1 / r)-\left(1+\varrho^{k}\right)}, \\
\Re\left[\frac{z B^{\prime}(z)}{B(z)}\right] & >1-\sum_{k=1}^{n} \frac{r\left(1-\varrho_{k}^{2}\right)}{\left(1-r \varrho_{k}\right)\left(\varrho_{k}-r\right)} .
\end{aligned}
$$

Thus

$$
\begin{aligned}
& 1-\sum_{k=1}^{n} \frac{r\left(1-\varrho_{k}^{2}\right)}{\left(1-r \varrho_{k}\right)\left(\varrho_{k}-r\right)}=1-r \sum_{k=1}^{n} \frac{\left(1-\varrho_{k}^{2}\right)\left(1 / \varrho^{k}\right)}{\left(1-r \varrho_{k}\right)\left(1-\varrho_{k} r\right)} \\
& \quad \geq 1-r \sum_{k=1}^{n} \frac{\left(1-\varrho_{k}^{2}\right)\left(1 / \varrho^{k}\right)}{\left(1-r \varrho_{1}\right)\left(1-\varrho_{k} r\right)}=1-r \sum_{k=1}^{n} \frac{\left(1-\varrho_{k}^{2}\right)\left(1 / \varrho_{k}^{2}\right)}{\left(1-r / \varrho_{1}\right)\left(1 / \varrho_{k}-r\right)} \\
& \quad \geq 1-r \sum_{k=1}^{n} \frac{\left(1-\varrho_{k}^{2}\right)\left(1 / \varrho_{k}^{2}\right)}{\left(1-r / \varrho_{1}\right)\left(1 / \varrho_{n}-r\right)}=1-r \sum_{k=1}^{n} \frac{\left(1-\varrho_{k}^{2}\right)\left[G\left(\varrho_{1}, \varrho_{n}\right) / \varrho_{k}\right]^{2}}{\left(\varrho_{1}-r\right)\left(1-\varrho_{n} r\right)}
\end{aligned}
$$

where $G\left(\varrho_{1}, \varrho_{n}\right)=\sqrt{\varrho_{1} \varrho_{n}}$. Define

$$
\Sigma=\sum_{k=1}^{n}\left(1-\varrho_{k}^{2}\right)\left[\frac{G\left(\varrho_{1}, \varrho_{n}\right)}{\varrho_{k}}\right]^{2}>0
$$

and note $\Sigma$ is not a function of $r$. The function

$$
g(r)=\frac{r \Sigma}{\left(1-r \varrho_{n}\right)\left(\varrho_{1}-r\right)}
$$

increases continuously from 0 to $+\infty$ on the interval $\left(0, \varrho_{1}\right)$. One finds that the value $r^{*}$ for which $1-g(r) \geq 0$ when $0 \leq r<r^{*}<\varrho_{1}$ is

$$
r^{*}=\frac{2 \varrho_{1}}{1+G^{2}+\Sigma+\sqrt{\left[(1+G)^{2}+\Sigma\right]\left[(1-G)^{2}+\Sigma\right]}} .
$$

The result then follows.

Notes. 1. $r^{*}$ is seen to depend on the geometric mean of $\varrho_{1}$ and $\varrho_{n}$ and the set $\left\{G / \varrho_{k}\right\}_{k=1}^{n}$, which measures the placement of the $\varrho_{k}$ relative to $G$. 
2. Routine limit calculations show that if

$$
B(z)=z \prod_{k=1}^{\infty} \frac{\left|z_{k}\right|}{z_{k}} \frac{z-z_{k}}{1-\bar{z}_{k} z},
$$

then $B$ is starlike on $r<r^{* *}$ where $r^{* *}=2 \varrho_{1} /\left(A_{\infty}+\sqrt{B_{\infty}}\right)$ with

$$
\begin{aligned}
& A_{\infty}:=1+G^{2}+\sum_{k=1}^{\infty}\left(1-\varrho_{k}^{2}\right)\left(\frac{G}{\varrho_{k}}\right)^{2}, \\
& B_{\infty}:=\left[(1+G)^{2}+\sum_{k=1}^{\infty}\left(1-\varrho_{k}^{2}\right)\left(\frac{G}{\varrho_{k}}\right)^{2}\right]\left[(1-G)^{2}+\sum_{k=1}^{\infty}\left(1-\varrho_{k}^{2}\right)\left(\frac{G}{\varrho_{k}}\right)^{2}\right] .
\end{aligned}
$$

\section{References}

[1] J. W. Alexander II, Functions which map the interior of the unit disc upon simple regions, Ann. of Math. 17 (1915), 12-22.

[2] A. Gluchoff and F. Hartmann, On a "much underestimated" paper of Alexander, Arch. Hist. Exact Sci. 55 (2000), 1-41.

[3] - , - A note on spiral-like polynomials, Sci. Math. Japon. 64 (2006), 517-523.

Mathematical Sciences

Villanova University

Villanova, PA 19085, U.S.A.

E-mail: alan.gluchoff@villanova.edu

frederick.hartmann@villanova.edu

Received 6.11.2007

and in final form 3.1.2008 